\title{
Immature Basophil Count
}

National Cancer Institute

\section{Source}

National Cancer Institute. Immature Basophil Count. NCI Thesaurus. Code C96670.

The determination of the amount of immature basophils present in a sample. 